\title{
Design, Synthesis, Molecular Docking, and Antibacterial Evaluation of Some Novel Flouroquinolone Derivatives as Potent Antibacterial Agent
}

\author{
Mehul M. Patel ${ }^{1}$ and Laxman J. Patel ${ }^{2}$ \\ ${ }^{1}$ Ramanbhai Patel College of Pharmacy, Charotar University of Science and Technology, CHARUSAT Campus, Changa, Petlad Taluka, \\ Anand District, Gujarat 388 421, India \\ ${ }^{2}$ S. K. Patel College of Pharmaceutical Education and Research, Ganpat University, Kherva, Gujarat 382711, India
}

Correspondence should be addressed to Mehul M. Patel; mehulpatel.ph@charusat.ac.in

Received 11 July 2014; Revised 28 October 2014; Accepted 29 October 2014; Published 9 December 2014

Academic Editor: Alessandro Sacchetti

Copyright (C) 2014 M. M. Patel and L. J. Patel. This is an open access article distributed under the Creative Commons Attribution License, which permits unrestricted use, distribution, and reproduction in any medium, provided the original work is properly cited.

Objective. Quinolone moiety is an important class of nitrogen containing heterocycles widely used as key building blocks for medicinal agents. It exhibits a wide spectrum of pharmacophores and has bactericidal, antiviral, antimalarial, and anticancer activities. In view of the reported antimicrobial activity of various fluoroquinolones, the importance of the C-7 substituents is that they exhibit potent antimicrobial activities. Our objective was to synthesize newer quinolone analogues with increasing bulk at C-7 position of the main 6-fluoroquinolone scaffold to produce the target compounds which have potent antimicrobial activity. Methods. A novel series of 1-ethyl-6-fluoro-4-oxo-7-\{4-[2-(4-substituted phenyl)-2-(substituted)-ethyl]-1-piperazinyl\}-1,4dihydroquinoline-3-carboxylic acid derivatives were synthesized. To understand the interaction of binding sites with bacterial protein receptor, the docking study was performed using topoisomerase II DNA gyrase enzymes (PDB ID: 2XCT) by Schrodinger's Maestro program. In vitro antibacterial activity of the synthesized compounds was studied and the MIC value was calculated by the broth dilution method. Results. Among all the synthesized compounds, some compounds showed potent antimicrobial activity. The compound $8 \mathrm{~g}$ exhibited good antibacterial activity. Conclusion. This investigation identified the potent antibacterial agents against certain infections.

\section{Introduction}

In recent years microbial infections are associated with high rates of attributable morbidity and mortality. Infections caused by microbial species are common in immune compromised patients and have significant treatment costs and mortality. The increasing rate of bacterial resistance to clinical antimicrobial agents and its impact on treatment of infectious diseases have begun to present a unique problem throughout the world. Drug resistant, multiple drug resistant (MDR), and extensively drug resistant (XDR) infectious bacterial pathogens put a greater risk on the population at large due to the risk of pandemic illness. Increasing complication is the fact that many antibacterial agents can induce mutations and resistance, often by different mechanisms. Methicillin-resistant Staphylococcus aureus (MRSA) and vancomycin-resistant Enterococcus (VRE) are the most important infections caused by bacteria which have been found worldwide in hospitals $[1,2]$. The emerging resistance of some microbial species to some synthetic antimicrobial agents makes it necessary to continue the search for new antimicrobial agents. Quinolone moiety is an important class of nitrogen containing heterocycles widely used as key building blocks for medicinal agents. It exhibits a wide spectrum of pharmacophores and has bactericidal [3], antiviral [4], antimalarial [5], and anticancer [6] activities. Among these, flouroquinolone derivatives play an important role in the field of medicine. Fluoroquinolones are the most widely used antibacterial agents in modern therapy due to their broad spectrum and excellent oral bioavailability. In 
this work our interest is to study the antibacterial activity of novel 1-ethyl-6-fluoro-4-oxo-7-\{4-[2-(4-substituted-phenyl)2-(substituted)-ethyl]-1-piperazinyl $\}$-1,4-dihydroquinoline3 -carboxylic acid derivatives. The present investigation deals with the clinically isolated different Gram-positive and Gram-negative bacteria against synthesized compounds and most of the tested compounds act as potent antibacterial agents. To understand the interactions of tested compounds at active sites of protein receptors, topoisomerase II DNA gyrase enzymes (PDB ID: 2XCT) molecular docking studies were also performed and reported in this paper.

\section{Materials and Method}

Melting points $\left({ }^{\circ} \mathrm{C}\right.$, uncorrected) of all the synthesized compounds were checked in capillary tubes by using a digital melting point apparatus (Veego melting point apparatus). All the reactions were monitored by thin layer chromatography (TLC silica gel $0.25 \mathrm{~mm}, 60 \mathrm{G}$ F254; eluting solvents were chloroform : methanol : hexane $9: 0.5: 0.5)$. All the compounds were characterized by FT-IR spectrometer (IR Prestige-21, Shimadzu, Japan) using KBr pellets; ${ }^{1} \mathrm{HNMR}$ spectra were obtained in $\mathrm{CDCl}_{3}$ on Bruker AvanceII $400 \mathrm{MHz}$ instrument and chemical shifts were measured as parts per million downfield from tetramethylsilane (TMS) as internal standard. Mass spectra were recorded on LCMS 2010 EV S Shimadzu mass spectrometer.

2.1. Procedure for Synthesis of 3-Chloro-4-fluoro Ethyl Anilinomethylene Malonate [7] (2). A mixture of 3-chloro-4fluoro aniline (1) $(1.4 \mathrm{~g}, 10 \mathrm{mmol})$ and diethyl ethoxymethylene malonate $(2.16 \mathrm{~g}, 10 \mathrm{mmol})$ was taken in glass microwave vessel and kept in microwave at $80 \%$ intensity for $60 \mathrm{sec}(3$ times). Ethanol was removed by vacuum pump. The crude solid was dried and recrystallized from n-hexane (yield $90 \%$; melting point $55-56^{\circ} \mathrm{C}\left(55-57^{\circ} \mathrm{C}\right) ; R_{f}$ value 0.73 (mobile phase: hexane : ethyl acetate $2: 1)$ ).

2.2. Procedure for Synthesis of 7-Chloro-6-fluoro-1,4-dihydro4-oxoquinoline-3-carboxylic Acid Ethyl Ester [8] (3). The 3chloro-4-fluoro ethyl anilinomethylene malonate (2) (1 g, $3.17 \mathrm{mmol})$ was added to diphenyl ether $(10 \mathrm{~mL})$ and refluxed for 2 to $3 \mathrm{hrs}$. The mixture was cooled, filtered, and dried. The crude solid obtained was purified by recrystallization from hexane (yield $85 \%$; melting point $288-290^{\circ} \mathrm{C}\left(290^{\circ} \mathrm{C}\right)$; $R_{f}$ value 0.68 (mobile phase: chloroform : methanol 9:1)).

2.3. Procedure for Synthesis of 7-Chloro-1-ethyl-6-fluoro1,4-dihydro-4-oxoquinoline-3-carboxylic Acid Ethyl Ester [8] (4). The mixture of 7-chloro-6-fluoro-1,4-dihydro4-oxoquinoline-3-carboxylic acid ethyl ester (3) (0.50 g, $1.86 \mathrm{mmol})$, ethyl iodide $(1.45 \mathrm{~g}, 9.3 \mathrm{mmol})$, potassium carbonate $(6.42 \mathrm{mg}, 46.5 \mathrm{mmol})$, and dimethyl formamide $(20 \mathrm{~mL})$ was taken in $50 \mathrm{~mL}$ round bottom flask and heated at $110-120^{\circ} \mathrm{C}$ for 5 to 6 hrs. Ice cold water was added to the reaction mixture. The crude solid obtained was purified and recrystallized from ethanol (yield $75 \%$; melting point $139-141^{\circ} \mathrm{C}\left(142^{\circ} \mathrm{C}\right) ; R_{f}$ value 0.93 (mobile phase: chloroform : methanol $9: 1)$ ).

2.4. Procedure for Synthesis of 7-Chloro-1-ethyl-6-fluoro-1,4dihydro-4-oxoquinoline-3-carboxylic Acid [8] (5). 7-Chloro1-ethyl-6-fluoro-1,4-dihydro-4-oxoquinoline-3-carboxylic acid ethyl ester (4) (200 mg, $0.674 \mathrm{mmol})$ and sodium hydroxide $(2 \mathrm{~N}, 2 \mathrm{~mL})$ were taken into $50 \mathrm{~mL}$ round bottom flask and refluxed for 2 to $3 \mathrm{hrs}$. The resulting mixture was filtered, filtrate was neutralized by acetic acid $(\mathrm{pH} 2)$, and separated solid was collected and washed with water. The solid was recrystallized with methanol (yield 62\%; melting point $280-282^{\circ} \mathrm{C}\left(284^{\circ} \mathrm{C}\right) ; R_{f}$ value 0.47 (mobile phase: chloroform : methanol $9: 1)$ ).

2.5. Procedure for Synthesis of 1-Ethyl-6-fluoro-1,4-dihydro-4oxo-7-(1-piperazinyl)-quinoline-3-carboxylic Acid [8] (6). In $50 \mathrm{~mL}$ glass microwave vessel, a mixture of $5(215.6 \mathrm{mg}$, $0.80 \mathrm{mmol})$, piperazine $(75.8 \mathrm{mg}, 0.88 \mathrm{mmol})$, triethylamine (165.6 mg, $2.40 \mathrm{mmol}$ ), and dimethyl formamide $(1 \mathrm{~mL})$ was irradiated in microwave at $80 \%$ intensity for a given interval of time $(60+60+30 \mathrm{sec})$. The mixture was evaporated to dryness. Water was added to the reaction mixture and the residue was filtered off, washed with water, dried, and recrystallized from dichloromethane and methanol to give $\mathbf{6}$ (yield 62\%; melting point $223-225^{\circ} \mathrm{C}\left(226^{\circ} \mathrm{C}\right) ; R_{f}$ value 0.38 (mobile phase: chloroform : methanol 2:3)).

2.6. General Procedure for Synthesis of 1-Ethyl-6-fluoro-4oxo-7-\{4-[2-(4-substituted phenyl)-2-(substituted)-ethyl]1-piperazinyl\}-1,4-dihydroquinoline-3-carboxylic Acids (8). A mixture of 1-ethyl-6-fluoro-4-oxo-7-(piperazin1-yl)-1,4-dihydroquinoline-3-carboxylic acid (1 mmol) (6), sodium bicarbonate (2 mmol), and 2-bromo-4(Substituted)acetophenone (1 mmol) in dimethyl formamide $(40 \mathrm{~mL})$ was stirred at room temperature for 3 to $6 \mathrm{hrs}$. The reaction mixture was poured into ice water $(50 \mathrm{~mL})$ [9]. The separated solid of 7 was collected by filteration, dried, and purified by recrystallization from methanolchloroform. Reaction of 7 with phenylhydrazine, hydrazine, hydroxylamine, semicarbazide, and methoxylamine hydrochloride, respectively, in ethanol with glacial acetic acid for 3 to $6 \mathrm{hrs}$ at $110-120^{\circ} \mathrm{C}$ gave the corresponding compounds (8a-8t) (shown in Scheme 1) in 45-74\% overall yield (shown in Table 1 ).

\subsection{Spectral Data}

2.7.1. 1-Ethyl-6-fluoro-4-oxo-7-\{4-[2-phenyl-2-(phenyl-hydrazono)-ethyl]-piperazin-1-yl\}-1,4-dihydro-quinoline-3-carboxylic Acid (8a). IR (KBr): 1629, $1718(\mathrm{C}=\mathrm{O}), 3049(\mathrm{C}-\mathrm{H}) \mathrm{cm}^{-1}$; ${ }^{1} \mathrm{H}$ NMR $\left(500 \mathrm{MHz}, \mathrm{CDCl}_{3}\right) \delta 1.58\left(\mathrm{t}, 3 \mathrm{H},-\mathrm{CH}_{3}\right), \delta 4.31(\mathrm{q}$, $\left.2 \mathrm{H},-\mathrm{CH}_{2}\right), \delta 2.84 \& \delta 3.38(\mathrm{~m}, 8 \mathrm{H}$, Piperazinyl), $\delta 3.87(\mathrm{~s}$, $\left.2 \mathrm{H}, \mathrm{N}-\mathrm{CH}_{2}\right), \delta 6.83-8.10(\mathrm{~m}, 12 \mathrm{H}$, Aromatic $\underline{\mathrm{H}}), \delta 8.69(\mathrm{~s}, 1 \mathrm{H}$, $\mathrm{C}=\mathrm{C}-\mathrm{H}), \delta 10.83(\mathrm{~s}, 1 \mathrm{H}, \mathrm{N}-\mathrm{NH}-\mathrm{Ph}), \delta 15.08(\mathrm{~s}, 1 \mathrm{H}, \mathrm{COOH})$; MASS (EI) $m / z$ : Calcd for $\mathrm{C}_{30} \mathrm{H}_{30} \mathrm{~F}_{1} \mathrm{~N}_{5} \mathrm{O}_{3}$ : 527 Found: 528 . 
<smiles>CC=CC(=O)OCC</smiles>

(1)

(2)

(3)<smiles>CCn1cc(C(=O)O)c(=O)c2cc(F)c(Cl)cc21</smiles>

(5)<smiles>CCCn1cc(C(=O)OCC)c(=O)c2cc(F)c(Cl)cc21</smiles>

(4)<smiles>CCn1cc(C(=O)O)c(=O)c2cc(F)c(N3CCNCC3)cc21</smiles>

(6)

Reagents and conditions:<smiles>[R1]c1ccc(C(=O)CN2CCCCC2)cc1</smiles>

(I) Diethyl ethoxymethylenemalonate, irradiated in microwave

(II) Diphenyl ether, 135 to $140^{\circ} \mathrm{C}$, reflux, $2-3 \mathrm{hr}$

(III) Potassium carbonate, DMF, $110^{\circ} \mathrm{C}, 1 \mathrm{~h}$, ethyl iodide, $110^{\circ} \mathrm{C}, 5-6 \mathrm{~h}$

(IV) Sodium hydroxide, reflux for 2-3 hrs, acetic acid

(V) Piperazine, triethylamine, DMF, irradiated in microwave

(VI) 2-Bromo-4-(substituted)acetophenone, DMF, stirring, RT, 3-6 hrs

(VII) Phenyl hydrazine or hydrazine or hydroxylamine hydrochloride and

glacial acetic acid or sodium acetate, absolute ethanol, reflux for 3-6 hrs,<smiles>[R]c1ccc(C(=[Y16])CN2CCN(c3cc4c(cc3F)c(=O)c(C(=O)O)cn4CC)CC2)cc1</smiles>

Scheme 1: Synthesis of 1-ethyl-6-fluoro-4-oxo-7-\{4-[2-(4-substituted phenyl)-2-(substituted)-ethyl]-1-piperazinyl\}-1,4-dihydroquinoline-3carboxylic acid.

2.7.2. 1-Ethyl-6-fluoro-4-oxo-7-[4-(2-phenyl-2-hydrazono-ethyl)-piperazin-1-yl]-4-1,4-dihydro-quinoline-3-carboxylic Acid (8b). IR (KBr): 1624, $1701(\mathrm{C}=\mathrm{O}), 3369(\mathrm{~N}-\mathrm{H}) \mathrm{cm}^{-1} ;{ }^{1} \mathrm{H}$ $\operatorname{NMR}\left(500 \mathrm{MHz}, \mathrm{CDCl}_{3}\right) \delta 1.25\left(\mathrm{t}, 3 \mathrm{H},-\mathrm{CH}_{3}\right), \delta 4.32(\mathrm{q}, 2 \mathrm{H}$, $-\mathrm{CH}_{2}$ ), $\delta 2.80 \& \delta 3.34(\mathrm{~m}, 8 \mathrm{H}$, Piperazin), $\delta 3.75(\mathrm{~s}, 2 \mathrm{H}$, $\left.\mathrm{N}-\mathrm{CH}_{2}\right), \delta 6.87-8.09(\mathrm{~m}, 7 \mathrm{H}$, Aromatic $\mathrm{H}), \delta 8.68(\mathrm{~s}, 1 \mathrm{H}$, $\mathrm{C}=\mathrm{C}-\mathrm{H}), \delta 15.09$ (s, $1 \mathrm{H}, \mathrm{COOH})$; MASS (EI) $m / z$ : Calcd for $\mathrm{C}_{24} \mathrm{H}_{26} \mathrm{~F}_{1} \mathrm{~N}_{5} \mathrm{O}_{3}$ : 451 Found: 452.

2.7.3. 1-Ethyl-6-fluoro-4-oxo-7-[4-(2-phenyl-2-hydroxy-iminoethyl)-piperazin-1-yl]-1,4-dihydro-quinoline-3-carboxylic Acid (8c). IR (KBr): 1629, $1896(\mathrm{C}=\mathrm{O}), 3221(\mathrm{O}-\mathrm{H}) ;{ }^{1} \mathrm{H}$ $\operatorname{NMR}\left(500 \mathrm{MHz}, \mathrm{CDCl}_{3}\right) \delta 1.58\left(\mathrm{t}, 3 \mathrm{H},-\mathrm{CH}_{3}\right), \delta 4.31(\mathrm{q}, 2 \mathrm{H}$,
$-\mathrm{CH}_{2}$ ), $\delta 2.77 \& \delta 3.36$ (m, 8H, Piperazinyl), $\delta 3.88$ (s, $2 \mathrm{H}$, $\left.\mathrm{N}-\mathrm{CH}_{2}\right), \delta 6.80-8.10(\mathrm{~m}, 7 \mathrm{H}$, Aromatic $\underline{\mathrm{H}}), \delta 8.69(\mathrm{~s}, 1 \mathrm{H}$, $\mathrm{C}=\mathrm{C}-\mathrm{H}), \delta 15.08(\mathrm{~s}, 1 \mathrm{H}, \mathrm{COOH}) ;$ MASS $(\mathrm{EI}) \mathrm{m} / z$ : Calcd for $\mathrm{C}_{24} \mathrm{H}_{25} \mathrm{~F}_{1} \mathrm{~N}_{4} \mathrm{O}_{4}$ : 452 Found: 453 .

2.7.4. 1-Ethyl-6-fluoro-4-oxo-7-[4-(2-phenyl-2-carbamoylhydrazinylidene-ethyl)-piperazin-1-yl]-1,4-dihydro-quinoline3-carboxylic Acid (8d). IR ( $\mathrm{KBr}): 1685(\mathrm{C}=\mathrm{O}), 3037$ (C$\mathrm{H}) \mathrm{cm}^{-1} ;{ }^{1} \mathrm{H}$ NMR $\left(500 \mathrm{MHz}, \mathrm{CDCl}_{3}\right) \delta 4.25\left(\mathrm{q}, 2 \mathrm{H},-\mathrm{CH}_{2}\right)$ $\delta 2.72 \& \delta 3.32\left(\mathrm{~m}, 8 \mathrm{H}\right.$, Piperazinyl), $\delta 3.74\left(\mathrm{~s}, 2 \mathrm{H}, \mathrm{N}-\mathrm{CH}_{2}\right)$, $\delta$ 6.79-8.04 (m, 7H, Aromatic $\underline{\mathrm{H}}), \delta 8.63(\mathrm{~s}, 1 \mathrm{H}, \mathrm{C}=\mathrm{C}-\mathrm{H}), \delta$ 10.95 (s, $1 \mathrm{H},-\mathrm{N}-\mathrm{NH}-\mathrm{CO}-), \delta 14.97(\mathrm{~s}, 1 \mathrm{H}, \mathrm{COOH})$; MASS (EI) $m / z$ : Calcd for $\mathrm{C}_{25} \mathrm{H}_{27} \mathrm{~F}_{1} \mathrm{~N}_{6} \mathrm{O}_{4}$ : 494 Found: 495. 
TABLE 1: Physical data of synthesis of 1-ethyl-6-fluoro-4-oxo-7-\{4-[2-(4-substituted phenyl)-2-(substituted)-ethyl]-1-piperazinyl\}-1,4dihydroquinoline-3-carboxylic acid (8a-8t).<smiles></smiles>

(8)

\begin{tabular}{|c|c|c|c|c|c|c|c|c|}
\hline Sr. number & Comp. number & $\mathrm{R}_{1}$ & $\mathrm{R}_{2}$ & Mol. wt. & Molecular formula & Yield (\%) & Melting point $\left({ }^{\circ} \mathrm{C}\right)$ & $R_{f}{ }^{*}$ \\
\hline 1 & $8 a$ & $-\mathrm{H}$ & $-\mathrm{NHC}_{6} \mathrm{H}_{5}$ & 527 & $\mathrm{C}_{30} \mathrm{H}_{30} \mathrm{~F}_{1} \mathrm{~N}_{5} \mathrm{O}_{3}$ & 72 & $189-191$ & 0.66 \\
\hline 2 & $8 b$ & $-\mathrm{H}$ & $-\mathrm{NH}_{2}$ & 451 & $\mathrm{C}_{24} \mathrm{H}_{26} \mathrm{~F}_{1} \mathrm{~N}_{5} \mathrm{O}_{3}$ & 50 & $204-206$ & 0.39 \\
\hline 3 & $8 \mathrm{c}$ & $-\mathrm{H}$ & $-\mathrm{OH}$ & 452 & $\mathrm{C}_{24} \mathrm{H}_{25} \mathrm{~F}_{1} \mathrm{~N}_{4} \mathrm{O}_{4}$ & 64 & 201-203 & 0.37 \\
\hline 4 & $8 d$ & $-\mathrm{H}$ & $-\mathrm{NHCONH}_{2}$ & 494 & $\mathrm{C}_{25} \mathrm{H}_{27} \mathrm{~F}_{1} \mathrm{~N}_{6} \mathrm{O}_{4}$ & 54 & $252-254$ & 0.41 \\
\hline 5 & $8 e$ & $-\mathrm{H}$ & $-\mathrm{OCH}_{3}$ & 466 & $\mathrm{C}_{25} \mathrm{H}_{27} \mathrm{FN}_{4} \mathrm{O}_{4}$ & 60 & $231-234$ & 0.54 \\
\hline 6 & $8 f$ & $-\mathrm{OCH}_{3}$ & $-\mathrm{NHC}_{6} \mathrm{H}_{5}$ & 557 & $\mathrm{C}_{31} \mathrm{H}_{32} \mathrm{~F}_{1} \mathrm{~N}_{5} \mathrm{O}_{4}$ & 70 & $228-230$ & 0.60 \\
\hline 7 & $8 g$ & $-\mathrm{OCH}_{3}$ & $-\mathrm{NH}_{2}$ & 481 & $\mathrm{C}_{25} \mathrm{H}_{28} \mathrm{~F}_{1} \mathrm{~N}_{5} \mathrm{O}_{4}$ & 60 & $160-162$ & 0.39 \\
\hline 8 & $8 h$ & $-\mathrm{OCH}_{3}$ & $-\mathrm{OH}$ & 482 & $\mathrm{C}_{25} \mathrm{H}_{27} \mathrm{~F}_{1} \mathrm{~N}_{4} \mathrm{O}_{5}$ & 55 & $215-218$ & 0.35 \\
\hline 9 & $8 \mathbf{i}$ & $-\mathrm{OCH}_{3}$ & $-\mathrm{NHCONH}_{2}$ & 524 & $\mathrm{C}_{26} \mathrm{H}_{29} \mathrm{~F}_{1} \mathrm{~N}_{6} \mathrm{O}_{5}$ & 62 & $242-245$ & 0.41 \\
\hline 10 & $8 \mathbf{j}$ & $-\mathrm{OCH}_{3}$ & $-\mathrm{OCH}_{3}$ & 496 & $\mathrm{C}_{26} \mathrm{H}_{29} \mathrm{FN}_{4} \mathrm{O}_{5}$ & 61 & $235-238$ & 0.54 \\
\hline 11 & $8 k$ & $-\mathrm{CH}_{3}$ & $-\mathrm{NHC}_{6} \mathrm{H}_{5}$ & 541 & $\mathrm{C}_{31} \mathrm{H}_{32} \mathrm{~F}_{1} \mathrm{~N}_{5} \mathrm{O}_{3}$ & 64 & $227-230$ & 0.62 \\
\hline 12 & 81 & $-\mathrm{CH}_{3}$ & $-\mathrm{NH}_{2}$ & 465 & $\mathrm{C}_{25} \mathrm{H}_{28} \mathrm{~F}_{1} \mathrm{~N}_{5} \mathrm{O}_{3}$ & 53 & $198-201$ & 0.37 \\
\hline 13 & $8 \mathrm{~m}$ & $-\mathrm{CH}_{3}$ & $-\mathrm{OH}$ & 466 & $\mathrm{C}_{25} \mathrm{H}_{27} \mathrm{~F}_{1} \mathrm{~N}_{4} \mathrm{O}_{4}$ & 58 & $242-244$ & 0.35 \\
\hline 14 & $8 n$ & $-\mathrm{CH}_{3}$ & $-\mathrm{NHCONH}_{2}$ & 508 & $\mathrm{C}_{26} \mathrm{H}_{29} \mathrm{~F}_{1} \mathrm{~N}_{6} \mathrm{O}_{4}$ & 61 & $158-160$ & 0.43 \\
\hline 15 & 80 & $-\mathrm{CH}_{3}$ & $-\mathrm{OCH}_{3}$ & 480 & $\mathrm{C}_{26} \mathrm{H}_{29} \mathrm{~F}_{1} \mathrm{~N}_{4} \mathrm{O}_{4}$ & 65 & 202-204 & 0.58 \\
\hline 16 & $8 p$ & $-\mathrm{NO}_{2}$ & $-\mathrm{NHC}_{6} \mathrm{H}_{5}$ & 572 & $\mathrm{C}_{30} \mathrm{H}_{29} \mathrm{FN}_{6} \mathrm{O}_{5}$ & 54 & $228-230$ & 0.62 \\
\hline 17 & $8 q$ & $-\mathrm{NO}_{2}$ & $-\mathrm{NH}_{2}$ & 496 & $\mathrm{C}_{24} \mathrm{H}_{25} \mathrm{FN}_{6} \mathrm{O}_{5}$ & 62 & $177-180$ & 0.58 \\
\hline 18 & $8 r$ & $-\mathrm{NO}_{2}$ & $-\mathrm{OH}$ & 497 & $\mathrm{C}_{24} \mathrm{H}_{24} \mathrm{FN}_{5} \mathrm{O}_{6}$ & 69 & $258-260$ & 0.52 \\
\hline 19 & $8 s$ & $-\mathrm{NO}_{2}$ & $-\mathrm{NHCONH}_{2}$ & 539 & $\mathrm{C}_{25} \mathrm{H}_{26} \mathrm{FN}_{7} \mathrm{O}_{6}$ & 72 & $207-209$ & 0.58 \\
\hline 20 & $8 t$ & $-\mathrm{NO}_{2}$ & $-\mathrm{OCH}_{3}$ & 511 & $\mathrm{C}_{25} \mathrm{H}_{26} \mathrm{FN}_{5} \mathrm{O}_{6}$ & 70 & $230-232$ & 0.62 \\
\hline
\end{tabular}

${ }^{*}$ Mobile phase: chloroform : hexane : methanol $(9: 0.5: 0.5)$.

2.7.5. 1-Ethyl-6-fluoro-4-oxo-7-[4-(2-phenyl-2-methoxy-iminoethyl)-piperazin-1-yl]-1,4-dihydro-quinoline-3-carboxylic Acid (8e). IR (KBr): 1633, $1718(\mathrm{C}=\mathrm{O}), 2937(\mathrm{C}-\mathrm{H}) \mathrm{cm}^{-1} ;{ }^{1} \mathrm{H}$ $\operatorname{NMR}\left(500 \mathrm{MHz}, \mathrm{CDCl}_{3}\right) \delta 4.25\left(\mathrm{q}, 2 \mathrm{H},-\mathrm{CH}_{2}\right) \delta 2.72 \& \delta$ $3.28\left(\mathrm{~m}, 8 \mathrm{H}\right.$, Piperazinyl), $\delta 3.70\left(\mathrm{~s}, 2 \mathrm{H}, \mathrm{N}-\mathrm{CH}_{2}\right), \delta 6.77-8.27$ $(\mathrm{m}, 7 \mathrm{H}$, Aromatic $\underline{\mathrm{H}}), \delta 8.61(\mathrm{~s}, 1 \mathrm{H}, \mathrm{C}=\mathrm{C}-\mathrm{H}), \delta 14.97(\mathrm{~s}$, $1 \mathrm{H}, \mathrm{COOH}$ ); MASS (EI) $m / z$ : Calcd for $\mathrm{C}_{25} \mathrm{H}_{27} \mathrm{FN}_{4} \mathrm{O}_{4}: 466$ Found: 467.

2.7.6. 1-Ethyl-6-fluoro-4-oxo-7-\{4-[2-(4-methoxyphenyl)-2(phenyl-hydrazono)-ethyl]-piperazin-1-yl\}-1,4-dihydro-quinoline-3-carboxylic Acid (8f). IR (KBr): 1625, $1728(\mathrm{C}=\mathrm{O})$, $3057(\mathrm{C}-\mathrm{H}) \mathrm{cm}^{-1} ;{ }^{1} \mathrm{H}$ NMR $\left(500 \mathrm{MHz}, \mathrm{CDCl}_{3}\right) \delta 1.58(\mathrm{t}$, $\left.3 \mathrm{H},-\mathrm{CH}_{3}\right), \delta 4.32\left(\mathrm{q}, 2 \mathrm{H},-\mathrm{CH}_{2}\right), \delta 2.83 \& \delta 3.37(\mathrm{~m}, 8 \mathrm{H}$, Piperazinyl), $\delta 3.83\left(\mathrm{~s}, 2 \mathrm{H}, \mathrm{N}-\mathrm{CH}_{2}\right), \delta 3.84\left(\mathrm{~s}, 3 \mathrm{H},-\mathrm{OCH}_{3}\right)$, $\delta$ 6.82-8.10 (m, 11H, Aromatic $\underline{\mathrm{H}}), \delta 8.68(\mathrm{~s}, 1 \mathrm{H}, \mathrm{C}=\mathrm{C}-\mathrm{H}), \delta$
10.69 (s, 1H, N-NH-Ph) $\delta 15.07$ (s, 1H, COOH); MASS (EI) $m / z$ : Calcd for $\mathrm{C}_{31} \mathrm{H}_{32} \mathrm{~F}_{1} \mathrm{~N}_{5} \mathrm{O}_{4}$ : 557 Found: 558 .

2.7.7. 1-Ethyl-6-fluoro-4-oxo-7-[4-(2-(4-methoxyphenyl)-2hydrazono-ethyl)-piperazin-1-yl]-4-1,4-dihydro-quinoline3-carboxylic Acid (8g). IR (KBr): 1624, 1718 (C=O), 3408 $(\mathrm{N}-\mathrm{H}) \mathrm{cm}^{-1} ;{ }^{1} \mathrm{H}$ NMR $\left(500 \mathrm{MHz}, \mathrm{CDCl}_{3}\right) \delta 4.31(\mathrm{q}, 2 \mathrm{H}$, $\left.-\mathrm{CH}_{2}\right) \delta 2.85 \& \delta 3.34(\mathrm{~m}, 8 \mathrm{H}$, Piperazinyl), $\delta 3.72(\mathrm{~s}$, $\left.2 \mathrm{H}, \mathrm{N}-\mathrm{CH}_{2}\right), \delta 3.83\left(\mathrm{~s}, 3 \mathrm{H},-\mathrm{OCH}_{3}\right), \delta 6.74-8.09(\mathrm{~m}, 6 \mathrm{H}$, Aromatic $\underline{\mathrm{H}}), \delta 8.68(\mathrm{~s}, 1 \mathrm{H}, \mathrm{C}=\mathrm{C}-\mathrm{H}), \delta 15.09(\mathrm{~s}, 1 \mathrm{H}, \mathrm{COOH})$; MASS (EI) $m / z$ : Calcd for $\mathrm{C}_{25} \mathrm{H}_{28} \mathrm{~F}_{1} \mathrm{~N}_{5} \mathrm{O}_{4}$ : 481 Found: 482.

2.7.8. 1-Ethyl-6-fluoro-4-oxo-7-[4-(2-(4-methoxyphenyl)-2hydroxy-iminoethyl)-piperazin-1-yl]-1,4-dihydro-quinoline3-carboxylic Acid (8h). IR (KBr): 1695, 1716 (C=O), 3271 $(\mathrm{OH}) \mathrm{cm}^{-1} ;{ }^{1} \mathrm{H}$ NMR $\left(500 \mathrm{MHz}, \mathrm{CDCl}_{3}\right) \delta 4.31(\mathrm{q}, 2 \mathrm{H}$, $\left.-\mathrm{CH}_{2}\right) \delta 2.75 \& \delta 3.35(\mathrm{~m}, 8 \mathrm{H}$, Piperazinyl), $\delta 3.88(\mathrm{~s}$, 
$\left.2 \mathrm{H}, \mathrm{N}-\mathrm{CH}_{2}\right), \delta 3.84\left(\mathrm{~s}, 3 \mathrm{H},-\mathrm{OCH}_{3}\right), \delta 6.90-8.11(\mathrm{~m}, 6 \mathrm{H}$, Aromatic $\underline{\mathrm{H}}), \delta 8.69(\mathrm{~s}, 1 \mathrm{H}, \mathrm{C}=\mathrm{C}-\mathrm{H}), \delta 15.08(\mathrm{~s}, 1 \mathrm{H}, \mathrm{COOH})$; MASS (EI) $m / z$ : Calcd for $\mathrm{C}_{25} \mathrm{H}_{27} \mathrm{~F}_{1} \mathrm{~N}_{4} \mathrm{O}_{5}$ : 482 Found: 483.

2.7.9. 1-Ethyl-6-fluoro-4-oxo-7-[4-(2-(4-methoxyphenyl)-2carbamoylhydrazinylidene-ethyl)-piperazin-1-yl]-1,4-dihydroquinoline-3-carboxylic Acid (8i). IR (KBr): 1674, $1720(\mathrm{C}=\mathrm{O})$, $3016,3485(\mathrm{~N}-\mathrm{H}) \mathrm{cm}^{-1} ;{ }^{1} \mathrm{H}$ NMR $\left(500 \mathrm{MHz}, \mathrm{CDCl}_{3}\right) \delta 4.33$ $\left(\mathrm{q}, 2 \mathrm{H},-\mathrm{CH}_{2}\right), \delta 2.83 \& \delta 3.31(\mathrm{~m}, 8 \mathrm{H}$, Piperazinyl), $\delta 3.71$ $\left(\mathrm{s}, 2 \mathrm{H}, \mathrm{N}-\mathrm{CH}_{2}\right), \delta 3.83\left(\mathrm{~s}, 3 \mathrm{H},-\mathrm{OCH}_{3}\right), \delta 6.77-8.09(\mathrm{~m}, 6 \mathrm{H}$, Aromatic $\underline{\mathrm{H}}), \delta 8.67$ (s, $1 \mathrm{H}, \mathrm{C}=\mathrm{C}-\mathrm{H}), \delta 15.05$ (s, 1H, COOH); MASS (EI) $m / z$ : Calcd for $\mathrm{C}_{26} \mathrm{H}_{29} \mathrm{~F}_{1} \mathrm{~N}_{6} \mathrm{O}_{5}$ : 524 Found: 525.

2.7.10. 1-Ethyl-6-fluoro-4-oxo-7-[4-(2-(4-methoxyphenyl)-2methoxy-iminoethyl)-piperazin-1-yl]-1,4-dihydro-quinoline3-carboxylic Acid (8j). IR (KBr): 1625, 1718 (C=O), 2939 $(\mathrm{C}-\mathrm{H}) \mathrm{cm}^{-1} ;{ }^{1} \mathrm{H} \mathrm{NMR}\left(500 \mathrm{MHz}, \mathrm{CDCl}_{3}\right) \delta 4.21(\mathrm{q}, 2 \mathrm{H}$, $\left.-\mathrm{CH}_{2}\right) \delta 2.66 \& \delta 3.21(\mathrm{~m}, 8 \mathrm{H}$, Piperazinyl), $\delta 3.71(\mathrm{~s}, 2 \mathrm{H}$, $\left.\mathrm{N}-\mathrm{CH}_{2}\right), \delta 3.94\left(\mathrm{~s}, 3 \mathrm{H},-\mathrm{OCH}_{3}\right.$ Side Chain) $\delta 3.85(\mathrm{~s}, 3 \mathrm{H}$, $\left.-\mathrm{OCH}_{3}\right), \delta 6.73-8.01(\mathrm{~m}, 7 \mathrm{H}$, Aromatic $\underline{\mathrm{H}}), \delta 8.60(\mathrm{~s}, 1 \mathrm{H}$, $\mathrm{C}=\mathrm{C}-\mathrm{H}), \delta 14.93(\mathrm{~s}, 1 \mathrm{H}, \mathrm{COOH})$; MASS $(\overline{\mathrm{EI}}) \mathrm{m} / z$ : Calcd for $\mathrm{C}_{26} \mathrm{H}_{29} \mathrm{FN}_{4} \mathrm{O}_{5}$ : 496 Found: 497.

2.7.11. 1-Ethyl-6-fluoro-4-oxo-7-\{4-[2-(4-methylphenyl)-2(phenyl-hydrazono)-ethyl]-piperazin-1-yl\}-1,4-dihydro-quinoline-3-carboxylic Acid (8k). IR (KBr): 1629, $1730(\mathrm{C}=\mathrm{O})$, $3062(\mathrm{C}-\mathrm{H}) \mathrm{cm}^{-1} ;{ }^{1} \mathrm{H}$ NMR $\left(500 \mathrm{MHz}, \mathrm{CDCl}_{3}\right) \delta 1.58(\mathrm{t}$, $\left.3 \mathrm{H},-\mathrm{CH}_{3}\right), \delta 4.32\left(\mathrm{q}, 2 \mathrm{H},-\mathrm{CH}_{2}\right), \delta 2.83 \& \delta 3.37(\mathrm{~m}, 8 \mathrm{H}$, Piperazinyl), $\delta 3.84\left(\mathrm{~s}, 2 \mathrm{H}, \mathrm{N}-\mathrm{CH}_{2}\right), \delta 2.38\left(\mathrm{~s}, 3 \mathrm{H},-\mathrm{CH}_{3}\right)$, $\delta$ 6.82-8.11 (m, 11H, Aromatic $\underline{\mathrm{H}}), \delta 8.68(\mathrm{~s}, 1 \mathrm{H}, \mathrm{C}=\mathrm{C}-\mathrm{H}), \delta$ $10.76(\mathrm{~s}, 1 \mathrm{H}, \mathrm{N}-\mathrm{NH}-\mathrm{Ph}) \delta 15.07$ (s, $1 \mathrm{H}, \mathrm{COOH}) ; \mathrm{MASS}$ (EI) $m / z$ : Calcd for $\mathrm{C}_{31} \mathrm{H}_{32} \mathrm{~F}_{1} \mathrm{~N}_{5} \mathrm{O}_{3}$ : 541 Found: 542 .

2.7.12. 1-Ethyl-6-fluoro-4-oxo-7-[4-(2-(4-methylphenyl)-2-hydrazono-ethyl)-piperazin-1-yl]-4-1,4-dihydro-quinoline-3carboxylic Acid (8l). IR (KBr): 1618, 1701 (C=O), 3387 $(\mathrm{N}-\mathrm{H}) \mathrm{cm}^{-1} ;{ }^{1} \mathrm{H}$ NMR $\left(500 \mathrm{MHz}, \mathrm{CDCl}_{3}\right) \delta 4.32(\mathrm{q}, 2 \mathrm{H}$, $\left.-\mathrm{CH}_{2}\right) \delta 2.78 \& \delta 3.33(\mathrm{~m}, 8 \mathrm{H}$, Piperazinyl), $\delta 3.72(\mathrm{~s}, 2 \mathrm{H}$, $\left.\mathrm{N}-\mathrm{CH}_{2}\right), \delta 2.39\left(\mathrm{~s}, 3 \mathrm{H},-\mathrm{CH}_{3}\right), \delta 6.80-8.09(\mathrm{~m}, 6 \mathrm{H}$, Aromatic $\underline{\mathrm{H}}), \delta 8.68(\mathrm{~s}, 1 \mathrm{H}, \mathrm{C}=\mathrm{C}-\mathrm{H}), \delta 15.07(\mathrm{~s}, 1 \mathrm{H}, \mathrm{COOH}) ; \mathrm{MASS}$ (EI) $m / z$ : Calcd for $\mathrm{C}_{25} \mathrm{H}_{28} \mathrm{~F}_{1} \mathrm{~N}_{5} \mathrm{O}_{3}$ : 465 Found: 466 .

2.7.13. 1-Ethyl-6-fluoro-4-oxo-7-[4-(2-(4-methylphenyl)-2-hydroxy-iminoethyl)-piperazin-1-yl]-1,4-dihydro-quinoline-3carboxylic Acid (8m). IR (KBr): 1629, $1718(\mathrm{C}=\mathrm{O}), 3277$ $(\mathrm{O}-\mathrm{H}) \mathrm{cm}^{-1} ;{ }^{1} \mathrm{H}$ NMR $\left(500 \mathrm{MHz}, \mathrm{CDCl}_{3}\right) \delta 4.31(\mathrm{q}, 2 \mathrm{H}$, $\left.-\mathrm{CH}_{2}\right) \delta 2.77 \& \delta 3.36(\mathrm{~m}, 8 \mathrm{H}$, Piperazinyl), $\delta 3.86(\mathrm{~s}, 2 \mathrm{H}$, $\left.\mathrm{N}-\mathrm{CH}_{2}\right), \delta 2.38\left(\mathrm{~s}, 3 \mathrm{H},-\mathrm{CH}_{3}\right), \delta 6.83-8.12(\mathrm{~m}, 6 \mathrm{H}$, Aromatic $\underline{\mathrm{H}}), \delta 8.69(\mathrm{~s}, 1 \mathrm{H}, \mathrm{C}=\mathrm{C}-\mathrm{H}), \delta 15.05(\mathrm{~s}, 1 \mathrm{H}, \mathrm{COOH}) ;$ MASS (EI) $m / z$ : Calcd for $\mathrm{C}_{25} \mathrm{H}_{27} \mathrm{~F}_{1} \mathrm{~N}_{4} \mathrm{O}_{4}$ : 466 Found: 467.

2.7.14. 1-Ethyl-6-fluoro-4-oxo-7-[4-(2-(4-methylphenyl)-2-carbamoylhydrazinylidene-ethyl)-piperazin-1-yl]-1,4-dihydroquinoline-3-carboxylic Acid (8n). IR (KBr): 1701, $1718(\mathrm{C}=\mathrm{O})$, $3032(\mathrm{C}-\mathrm{H}) \mathrm{cm}^{-1} ;{ }^{1} \mathrm{H}$ NMR $\left(500 \mathrm{MHz}, \mathrm{CDCl}_{3}\right) \delta 4.33(\mathrm{q}$, $\left.2 \mathrm{H},-\mathrm{CH}_{2}\right) \delta 2.78 \& \delta 3.35(\mathrm{~m}, 8 \mathrm{H}$, Piperazinyl $), \delta 3.13$ $\left(\mathrm{s}, 2 \mathrm{H}, \mathrm{N}-\mathrm{CH}_{2}\right), \delta 2.39\left(\mathrm{~s}, 3 \mathrm{H},-\mathrm{CH}_{3}\right), \delta 6.80-8.03(\mathrm{~m}$,
6H, Aromatic $\underline{\mathrm{H}}), \delta 8.68(\mathrm{~s}, 1 \mathrm{H}, \mathrm{C}=\mathrm{C}-\mathrm{H}), \delta 10.99(\mathrm{~s}, 1 \mathrm{H}$, -N-NH-CO-), $\delta 15.09$ (s, $1 \mathrm{H}, \mathrm{COOH}$ ); MASS (EI) $m / z$ : Calcd for $\mathrm{C}_{26} \mathrm{H}_{29} \mathrm{~F}_{1} \mathrm{~N}_{6} \mathrm{O}_{4}$ : 508 Found: 509.

2.7.15. 1-Ethyl-6-fluoro-4-oxo-7-[4-(2-(4-methylphenyl)-2-methoxy-iminoethyl)-piperazin-1-yl]-1,4-dihydro-quinoline-3carboxylic Acid (8o). IR (KBr): 1626, 1732 (C=O), 2937 $(\mathrm{C}-\mathrm{H}) \mathrm{cm}^{-1} ;{ }^{1} \mathrm{H} \mathrm{NMR}\left(500 \mathrm{MHz}, \mathrm{CDCl}_{3}\right) \delta 1.49(\mathrm{t}, 3 \mathrm{H}$, $\left.-\mathrm{CH}_{3}\right) \delta 4.22\left(\mathrm{q}, 2 \mathrm{H},-\mathrm{CH}_{2}\right), \delta 2.65 \& \delta 3.21(\mathrm{~m}, 8 \mathrm{H}$, Piperazinyl), $\delta 3.38$ (s, 2H, N- $\mathrm{CH}_{2}$ ), $\delta 3.91$ (s, 3H, $-\mathrm{OCH}_{3}$ Side Chain), $\delta 2.29\left(\mathrm{~s}, 3 \mathrm{H},-\mathrm{CH}_{3}\right), \delta 6.70-7.97(\mathrm{~m}, 6 \mathrm{H}$, Aromatic $\underline{\mathrm{H}}), \delta 8.58(\mathrm{~s}, 1 \mathrm{H}, \mathrm{C}=\mathrm{C}-\mathrm{H}), \delta 15.06(\mathrm{~s}, 1 \mathrm{H}, \mathrm{COOH})$; MASS (EI) $m / z$ : Calcd for $\mathrm{C}_{26} \mathrm{H}_{29} \mathrm{~F}_{1} \mathrm{~N}_{4} \mathrm{O}_{4}$ : 480 Found: 481 .

2.7.16. 1-Ethyl-6-fluoro-4-oxo-7-\{4-[2-(4-nitrophenyl)-2-(phenyl-hydrazono)-ethyl]-piperazin-1-yl\}-1,4-dihydro-quinoline-3-carboxylic Acid (8p). IR (KBr): 1629, 1707 ( $\mathrm{C}=\mathrm{O})$, $3336(\mathrm{~N}-\mathrm{H}) \mathrm{cm}^{-1} ;{ }^{1} \mathrm{H} \mathrm{NMR}\left(500 \mathrm{MHz}, \mathrm{CDCl}_{3}\right) \delta 4.23(\mathrm{q}, 2 \mathrm{H}$, $\left.-\mathrm{CH}_{2}\right) \delta 2.77 \& \delta 3.32(\mathrm{~m}, 8 \mathrm{H}$, Piperazinyl), $\delta 3.83$ (s, 2H, N$\left.\mathrm{CH}_{2}\right), \delta 2.38\left(\mathrm{~s}, 3 \mathrm{H},-\mathrm{CH}_{3}\right), \delta$ 6.76-8.17 (m, 10H, Aromatic $\underline{\mathrm{H}}), \delta 8.62(\mathrm{~s}, 1 \mathrm{H}, \mathrm{C}=\mathrm{C}-\mathrm{H}), \delta 11.08(\mathrm{~s}, 1 \mathrm{H}, \mathrm{N}-\mathrm{NH}-\mathrm{Ph}), \delta 14.93$ $\left(\mathrm{s}, 1 \mathrm{H}, \mathrm{COOH}\right.$ ); MASS (EI) $m / z$ : Calcd for $\mathrm{C}_{30} \mathrm{H}_{29} \mathrm{FN}_{6} \mathrm{O}_{5}: 572$ Found: 573.

2.7.17. 1-Ethyl-6-fluoro-4-oxo-7-[4-(2-(4-nitrophenyl)-2-hydrazono-ethyl)-piperazin-1-yl]-4-1,4-dihydro-quinoline-3-carboxylic Acid (8q). IR (KBr): 1691, 1726 (C=O), 3394 (N$\mathrm{H}) \mathrm{cm}^{-1} ;{ }^{1} \mathrm{H}$ NMR $\left(500 \mathrm{MHz}, \mathrm{CDCl}_{3}\right) \delta 4.22\left(\mathrm{q}, 2 \mathrm{H},-\mathrm{CH}_{2}\right)$ $\delta 2.67 \& \delta 3.21$ ( $\mathrm{m}, 8 \mathrm{H}$, Piperazinyl), $\delta 3.83$ (s, 2H, N- $\left.\mathrm{CH}_{2}\right), \delta$ $6.72-7.98(\mathrm{~m}, 6 \mathrm{H}$, Aromatic $\underline{\mathrm{H}}), \delta 8.58(\mathrm{~s}, 1 \mathrm{H}, \mathrm{C}=\mathrm{C}-\mathrm{H}), \delta 15.06$ $(\mathrm{s}, 1 \mathrm{H}, \mathrm{COOH}) ; \mathrm{MASS}(\mathrm{EI}) \mathrm{m} / z$ : Calcd for $\mathrm{C}_{24} \mathrm{H}_{25} \mathrm{FN}_{6} \mathrm{O}_{5}: 496$ Found: 497.

2.7.18. 1-Ethyl-6-fluoro-4-oxo-7-[4-(2-(4-nitrophenyl)-2-hydroxy-iminoethyl)-piperazin-1-yl]-1,4-dihydro-quinoline3-carboxylic Acid (8r). IR (KBr): 1631, 1714 (C=O), 3199 $(\mathrm{OH}) \mathrm{cm}^{-1} ;{ }^{1} \mathrm{H}$ NMR $\left(500 \mathrm{MHz}, \mathrm{CDCl}_{3}\right) \delta 4.21(\mathrm{q}, 2 \mathrm{H}$, $\left.-\mathrm{CH}_{2}\right) \delta 2.69 \& \delta 3.23(\mathrm{~m}, 8 \mathrm{H}$, Piperazinyl), $\delta 3.81(\mathrm{~s}, 2 \mathrm{H}$, $\left.\mathrm{N}-\mathrm{CH}_{2}\right), \delta 6.87-8.08(\mathrm{~m}, 6 \mathrm{H}$, Aromatic $\underline{\mathrm{H}}), \delta 8.58(\mathrm{~s}, 1 \mathrm{H}$, $\mathrm{C}=\mathrm{C}-\mathrm{H}), \delta 15.06(\mathrm{~s}, 1 \mathrm{H}, \mathrm{COOH}) ; \mathrm{MASS}(\mathrm{EI}) \mathrm{m} / z$ : Calcd for $\mathrm{C}_{24} \mathrm{H}_{24} \mathrm{FN}_{5} \mathrm{O}_{6}$ : 497 Found: 498.

2.7.19. 1-Ethyl-6-fluoro-4-oxo-7-[4-(2-(4-nitrophenyl)-2-carbamoylhydrazinylidene-ethyl)-piperazin-1-yl]-1,4-dihydroquinoline-3-carboxylic Acid (8s). IR (KBr): 1691, $1726(\mathrm{C}=\mathrm{O})$, $3062(\mathrm{C}-\mathrm{H}) \mathrm{cm}^{-1} ;{ }^{1} \mathrm{H} \mathrm{NMR}\left(500 \mathrm{MHz}, \mathrm{CDCl}_{3}\right) \delta 4.21(\mathrm{q}$, $\left.2 \mathrm{H},-\mathrm{CH}_{2}\right), \delta 2.67 \& \delta 3.26(\mathrm{~m}, 8 \mathrm{H}$, Piperazinyl), $\delta 3.84$ $\left(\mathrm{s}, 2 \mathrm{H}, \mathrm{N}-\mathrm{CH}_{2}\right), \delta 6.75-8.01(\mathrm{~m}, 6 \mathrm{H}$, Aromatic $\underline{\mathrm{H}}), \delta 8.57$ $(\mathrm{s}, 1 \mathrm{H}, \mathrm{C}=\mathrm{C}-\mathrm{H}), \delta 10.97$ (s, $1 \mathrm{H},-\mathrm{N}-\mathrm{NH}-\mathrm{CO}-), \delta 15.01$ (s, $1 \mathrm{H}, \mathrm{COOH}$ ); MASS (EI) $m / z$ : Calcd for $\mathrm{C}_{25} \mathrm{H}_{26} \mathrm{FN}_{7} \mathrm{O}_{6}: 539$ Found: 540.

2.7.20. 1-Ethyl-6-fluoro-4-oxo-7-[4-(2-(4-nitrophenyl)-2-methoxy-iminoethyl)-piperazin-1-yl]-1,4-dihydro-quinoline-3carboxylic Acid (8t). IR (KBr): 1629, 1722 (C=O), 2939 $(\mathrm{C}-\mathrm{H}) \mathrm{cm}^{-1} ;{ }^{1} \mathrm{H}$ NMR $\left(500 \mathrm{MHz}, \mathrm{CDCl}_{3}\right) \delta 1.50(\mathrm{t}, 3 \mathrm{H}$, $\left.-\mathrm{CH}_{3}\right) \delta 4.23\left(\mathrm{q}, 2 \mathrm{H},-\mathrm{CH}_{2}\right), \delta 2.67 \& \delta 3.21(\mathrm{~m}, 8 \mathrm{H}$, 
Piperazinyl), $\delta 3.39$ (s, 2H, N- $\mathrm{CH}_{2}$ ), $\delta 3.85$ (s, 3H, $-\mathrm{OCH}_{3}$ Side Chain), $\delta 6.72-8.20$ (m, $6 \mathrm{H}$, Aromatic $\underline{\mathrm{H}}), \delta 8.59(\mathrm{~s}, 1 \mathrm{H}$, $\mathrm{C}=\mathrm{C}-\mathrm{H}), \delta 15.03$ (s, $1 \mathrm{H}, \mathrm{COOH}$ ); MASS (EI) $\mathrm{m} / z$ : Calcd for $\mathrm{C}_{25} \mathrm{H}_{26} \mathrm{FN}_{5} \mathrm{O}_{6}$ : 511 Found: 512 .

2.8. Molecular Docking Studies. Molecular docking is an important computational technique in structural biology and computer aided drug design. The major objective of molecular docking is to evaluate the feasible binding geometries of a putative ligand with a target protein of known threedimensional structure.

To understand the interaction of all the synthesized molecules $(\mathbf{8 a}-\mathbf{8 t})$ with topoisomerase II DNA gyrase enzymes, the crystal structure of topoisomerase II was downloaded from the Protein Data Bank (PDB ID: 2XCT) and molecular docking studies were performed using the Glide program $[10,11]$ (version 6.3, Schrodinger, LLC, New York, 2014). To analyze the docking results and execute the protocol, the Maestro user interface (version 9.7, Schrodinger, LLC, New York, 2014) was employed. Docking was performed using the XP (Extra Precision Mode) docking protocol.

2.8.1. Preparation of Protein. The structure of topoisomerase II DNA gyrase enzyme (PDB ID: 2XCT) was downloaded from the Protein Data Bank and imported and prepared by a multistep process through the protein preparation wizard of Maestro (version 9.7). The protocol was especially used to obtain the optimised and minimised energy conformation of the protein. Firstly, the bond order in the protein was assigned; hydrogen atoms were added and the water molecules which did not participate in interactions were removed. Following the above steps of preparation, the protein was subjected to energy minimisation using Schrodinger implementation of OPLS-2005 force field with implicit solvation.

2.8.2. Preparation of Ligands. The ligands were prepared using the LigPrep 3.0 [12] module of the Schrodinger suite [13] using Merck molecular force field (MMFF). MMFF was used for $2 \mathrm{D}$ to $3 \mathrm{D}$ conversion of ligand molecules while the optimized potential for liquid simulation (OPLS) force field was used for conformation analysis through ConfGen [14].

2.9. Active Site Prediction. Active site is a pocket pouch present on the protein structure that has the tendency to accept the ligand molecules within it. The Sitemap applies theoretical methods and predicts the most accurate binding site. The OPLS-AA force field generates site points, possible for ligand interaction within the protein. Sitemap gives an idea about positions favorable for a donor, acceptor, and hydrophobic group to be present in the receptor. The maps were very useful for analysing the interactions of ligands with the receptor. In order to find the active site volume, surface area, and character of binding sites of the enzymes (2XCT), Sitemap was performed by choosing the top ranked potential receptor binding sites and the other parameters were kept as default. OPLS-2005 was used as the force field. Five sites with different site scores were obtained as output and the site with the highest site score was selected.

Docking studies on LigPrep treated ligands were carried out to predict the binding pocket of 2XCT using the docking program, Glide. Glide used a series of hierarchical filters to search for possible locations for the ligand in the active site region of the receptor. For the grid-based ligand docking, the receptor grid generation file was used. For protein structure, a grid box of $30 \times 30 \times 30 \AA$ with a default inner box $(10 \times 10 \times$ $10 \AA$ ) was centered on the corresponding ligand. The receptor grid was defined as an enclosing box at the centroid of the cocrystallized ligand (i.e., 2XCT) to include the cofactor and substrate binding sites. In the initial Glide docking stage, a softened potential docking with the van der Waals radii scaling of 0.7 for the proteins was performed to retain the maximum number of 20 poses per ligand. Residues within $5.0 \AA$ of ligand poses were kept free to move in the Prime refinement step, and the side chains were further minimized. Then single low energy 3D structure of ligands with correct chiralities was docked with the binding site using the "Extra Precision" Glide algorithm in Schrodinger.

2.10. In Vitro Antibacterial Activity. All the synthesized compounds were studied for their antibacterial activity against two clinically isolated Gram-positive strains ( $S$. aureus and $S$. pneumoniae) and two Gram-negative strains (P. aeruginosa and Escherichia coli) using conventional broth dilution method. The minimum inhibitory concentration (MIC) values were calculated by comparison to norfloxacin as the reference bacterial drug and they are shown in Table 2.

All the cultures were prepared by Mueller-Hinton agar and the turbidity of all the bacterial cultures was adjusted to $0.5 \mathrm{McF}$ arland standard by preparing bacterial suspension of 3-5 well-isolated colonies of the same morphological type selected from an agar plate culture. The cultures were further diluted 1000-fold to get an inoculum size of $1 \times 10^{6} \mathrm{CFU}$. The synthesized compounds and standard bacterial drugs (50 mg) were dissolved in dimethyl sulphoxide (DMSO) $(0.5 \mathrm{~mL})$ and the solution was diluted with water $(4.5 \mathrm{~mL})$ to get a stock solution of $10,000 \mathrm{mg} / \mathrm{L}$ of each compound. Further progressive double dilution with melted Mueller-Hinton agar was performed to obtain the required concentrations of 100 , $50,25,12.5,6.25$, and $1 \mu \mathrm{g} / \mathrm{mL}[15,16]$. To ensure that the solvent had no effect on the bacterial growth, a control test was performed with a test medium supplemented with DMSO at the same dilution as that used in the experiment. Incubation of the cultures was done at $37^{\circ} \mathrm{C}$ for $24 \mathrm{hrs}$ and comparison with blank was done in terms of turbidity which is due to microbial growth.

\section{Results and Discussion}

3.1. Chemistry. 1-Ethyl-6-fluoro-4-oxo-7-\{4-[2-(4-substituted phenyl)-2-oxoethyl]-1-piperazinyl $\}$-1,4-dihydroquinoline3-carboxylic acid (7) was prepared according to the literature 
TABLE 2: In vitro antibacterial activity of synthesized compounds against Gram-positive and Gram-negative bacteria (MICs in $\mu \mathrm{g} / \mathrm{mL})$.

\begin{tabular}{|c|c|c|c|c|c|c|c|}
\hline \multicolumn{8}{|c|}{ Minimum inhibitory concentrations $(\mu \mathrm{g} \backslash \mathrm{mL})$} \\
\hline Sr. number & Comp. number & $\mathrm{R}_{1}$ & $\mathrm{R}_{2}$ & $\begin{array}{c}\text { S. aureus } \\
\text { ATCC-29213 }\end{array}$ & $\begin{array}{l}\text { S. pneumonia } \\
\text { ATCC-49619 }\end{array}$ & $\begin{array}{l}\text { P. aeruginosa } \\
\text { ATCC- } 27853\end{array}$ & $\begin{array}{c}\text { E. coli } \\
\text { ATCC-25922 } \\
\end{array}$ \\
\hline 1 & $8 \mathbf{8 a}$ & $-\mathrm{H}$ & $-\mathrm{NHC}_{6} \mathrm{H}_{5}$ & 50 & 100 & 100 & 25 \\
\hline 2 & $8 \mathbf{b}$ & $-\mathrm{H}$ & $-\mathrm{NH}_{2}$ & 25 & 100 & 100 & 12.5 \\
\hline 3 & $8 \mathrm{c}$ & $-\mathrm{H}$ & $-\mathrm{OH}$ & 50 & 100 & 100 & 50 \\
\hline 4 & $8 d$ & $-\mathrm{H}$ & $-\mathrm{NHCONH}_{2}$ & 12.5 & 100 & 100 & 25 \\
\hline 5 & $8 \mathrm{e}$ & $-\mathrm{H}$ & $-\mathrm{OCH}_{3}$ & 50 & $>100$ & $>100$ & 50 \\
\hline 6 & $8 f$ & $-\mathrm{OCH}_{3}$ & $-\mathrm{NHC}_{6} \mathrm{H}_{5}$ & 25 & 100 & 100 & 25 \\
\hline 7 & $8 g$ & $-\mathrm{OCH}_{3}$ & $-\mathrm{NH}_{2}$ & 6.25 & 12.5 & 100 & 12.5 \\
\hline 8 & $8 \mathrm{~h}$ & $-\mathrm{OCH}_{3}$ & $-\mathrm{OH}$ & 6.25 & 50 & 100 & 12.5 \\
\hline 9 & $8 \mathrm{i}$ & $-\mathrm{OCH}_{3}$ & $-\mathrm{NHCONH}_{2}$ & 6.25 & 12.5 & 50 & 12.5 \\
\hline 10 & $8 \mathbf{j}$ & $-\mathrm{OCH}_{3}$ & $-\mathrm{OCH}_{3}$ & 50 & 100 & 100 & 50 \\
\hline 11 & $8 k$ & $-\mathrm{CH}_{3}$ & $-\mathrm{NHC}_{6} \mathrm{H}_{5}$ & 50 & 100 & $>100$ & 25 \\
\hline 12 & 81 & $-\mathrm{CH}_{3}$ & $-\mathrm{NH}_{2}$ & 12.5 & 25 & 100 & 25 \\
\hline 13 & $8 m$ & $-\mathrm{CH}_{3}$ & $-\mathrm{OH}$ & 50 & 100 & 100 & 50 \\
\hline 14 & $8 n$ & $-\mathrm{CH}_{3}$ & $-\mathrm{NHCONH}_{2}$ & 6.25 & 25 & 50 & 12.5 \\
\hline 15 & 80 & $-\mathrm{CH}_{3}$ & $-\mathrm{OCH}_{3}$ & 25 & 100 & $>100$ & 25 \\
\hline 16 & $8 p$ & $-\mathrm{NO}_{2}$ & $-\mathrm{NHC}_{6} \mathrm{H}_{5}$ & 50 & 100 & $>100$ & 50 \\
\hline 17 & $\mathbf{8 q}$ & $-\mathrm{NO}_{2}$ & $-\mathrm{NH}_{2}$ & 12.5 & 25 & 50 & 12.5 \\
\hline 18 & $8 r$ & $-\mathrm{NO}_{2}$ & $-\mathrm{OH}$ & 6.25 & 50 & 100 & 12.5 \\
\hline 19 & $8 s$ & $-\mathrm{NO}_{2}$ & $-\mathrm{NHCONH}_{2}$ & 6.25 & 12.5 & 50 & 6.25 \\
\hline 20 & $8 \mathrm{t}$ & $-\mathrm{NO}_{2}$ & $-\mathrm{OCH}_{3}$ & 25 & 100 & 100 & 25 \\
\hline 21 & & Norfloxacin & & 1 & 6.25 & 6.25 & 1 \\
\hline
\end{tabular}

method. Our synthetic route to target compounds $(\mathbf{8} \mathbf{a}-$ $\mathbf{8 t})$ is presented in Scheme 1. The 1-ethyl-6-fluoro-4-oxo-7\{4-[2-(4-substituted phenyl)-2-oxoethyl]-1-piperazinyl\}-1,4dihydroquinoline-3-carboxylic acid derivatives (7) react with various amines to form target compounds. The structures were characterized by spectral techniques. In general, in FTIR spectra, characteristic N-H stretching peaks near 3269$3415 \mathrm{~cm}^{-1}$ and $\mathrm{C}=\mathrm{O}$ stretching peaks near $1622-1732 \mathrm{~cm}^{-1}$ are shown. The ${ }^{1} \mathrm{H}-\mathrm{NMR}$ of synthesized derivatives showed multiple signals corresponding to resonance of fluoroquinolone protons, at two $\mathrm{m}, 8 \mathrm{H}$ in the region of $\delta 2.64$ to 3.83 corresponding to piperazine hydrogen. The proton of the N$\mathrm{CH}_{2}$ group exhibited a singlet in the region of $\delta 3.65$ to 3.91. Aromatic protons were resonating as multiple in the region of $\delta 6.8$ to 8.19 . The proton of $\mathrm{O}-\mathrm{H}$ of carboxylic acid exhibited a singlet in the region of $\delta 14.72$ to 15.09 . The proton of the $\mathrm{C}-\mathrm{H}$ (alkenyl) of quinolone ring exhibited a singlet in the region of $\delta 8.58$ to 8.80 . All the compounds were characterized by mass spectral analysis. Compounds show that $\mathrm{M}^{+}$peak corresponds to the molecular weight.

\subsection{Biology}

3.2.1. In Vitro Antibacterial Studies of Fluoroquinolone Derivatives. All the synthesized compounds were tested against two Gram-positive and two Gram-negative bacteria. All the compounds (8a-8t) exhibited good bacterial activity against Gram-positive bacteria.
Against Gram-Positive Strain. Compounds 8g, 8h, 8i, 8n, 8r, and $8 \mathbf{s}$ have shown antibacterial activity with MIC value in the range of $6.25-25 \mu \mathrm{g} / \mathrm{mL}$ against Gram-positive $S$. aureus and $S$. pneumonia. Compounds $\mathbf{8 1}$ and $\mathbf{8 q}$ have shown antibacterial activity with MIC value in the range of 12.5$25 \mu \mathrm{g} / \mathrm{mL}$ against Gram-positive S. aureus and S. pneumonia. The Remaining compounds have shown antibacterial activity with MIC value in the range of 25 to $\geq 100 \mu \mathrm{g} / \mathrm{mL}$ against Gram-positive S. aureus and S. pneumonia.

Against Gram-Negative Strain. Compounds $\mathbf{8 i}, \mathbf{8 n}, \mathbf{8 q}$, and 8s have shown potent antibacterial activity with MIC value in the range of $6.25-50 \mu \mathrm{g} / \mathrm{mL}$ against Gram-negative E. coli and $P$. aeruginosa. The remaining compounds have shown antibacterial activity with MIC value in the range of $12.5-$ $50 \mu \mathrm{g} / \mathrm{mL}$ against Gram-negative E. coli and $\geq 100 \mu \mathrm{g} / \mathrm{mL}$ against Gram-negative $P$. aeruginosa. It was observed that $\mathbf{8 i}$, $\mathbf{8 1}, \mathbf{8 n}, \mathbf{8 q}$, and $\mathbf{8 s}$ have shown potent antibacterial activity against Gram-positive S. aureus and S. pneumonia and against Gram-negative E. coli strains and P. aeruginosa.

In terms of structure activity relationship, results suggest that compounds having 4-nitro and 4-methoxy on phenyl ring at C-7 piperazinyl side chain exhibited the most potent antibacterial activity. It was also observed that compounds with imino nitrogen substituted with a semicarbazide or amine $\left(R_{2}=-\mathrm{NHCONH}_{2}\right.$ and $\left.\mathrm{NH}_{2}\right)$ exhibited potent antibacterial activity.

Compounds $\mathbf{8 g}, \mathbf{8 h}, \mathbf{8 i}, \mathbf{8 n}, \mathbf{8 r}, \mathbf{8 s}, \mathbf{8 1}$, and $\mathbf{8 q}$ were found to be active as antibacterial agents. The pharmacological data 
TABLE 3: Docking result of fluoroquinolones analogue.

\begin{tabular}{|c|c|c|c|c|c|c|c|}
\hline Sr. number & Title & Docking G-score & Glide energy & Glide Emodel & XP H-bond & XP LipophilicEvdW & XP Electro \\
\hline 1 & $8 g$ & -8.51 & -54.3846 & -105.214 & -2.68 & -4.84 & -0.99 \\
\hline 2 & $8 \mathrm{i}$ & -8.23 & -72.1348 & -119.918 & -1.09 & -6.14 & -1.01 \\
\hline 3 & $8 e$ & -8.22 & -70.9957 & -98.0172 & -1.95 & -4.99 & -1.27 \\
\hline 4 & $8 \mathrm{c}$ & -7.95 & -73.5718 & -104.847 & -1.83 & -4.96 & -1.16 \\
\hline 5 & $8 s$ & -7.83 & -69.62 & -117.139 & -1.89 & -5.67 & -1.27 \\
\hline 6 & $\mathbf{8 q}$ & -7.68 & -63.5086 & -106.854 & -2.3 & -4.98 & -1.4 \\
\hline 7 & $8 h$ & -7.56 & -74.8308 & -102.867 & -2.27 & -4.06 & -1.23 \\
\hline 8 & $8 n$ & -7.53 & -65.1719 & -95.4165 & -0.68 & -5.69 & -1.16 \\
\hline 9 & $8 a$ & -7.43 & -57.3206 & -84.8511 & -2.16 & -5.19 & -1.08 \\
\hline 10 & $8 b$ & -7.26 & -69.2937 & -97.1236 & -2.11 & -4.74 & -1.41 \\
\hline 11 & $8 r$ & -7.26 & -65.4352 & -109.856 & -0.81 & -4.92 & -1.54 \\
\hline 12 & $8 p$ & -6.84 & -62.2759 & -99.8452 & -0.68 & -3.76 & -2.4 \\
\hline 13 & $8 \mathbf{j}$ & -6.4 & -69.2569 & -108.236 & -1.76 & -4.42 & -1.21 \\
\hline 14 & $8 f$ & -6.39 & -69.32 & -107.169 & -0.64 & -6.27 & -0.49 \\
\hline 15 & $8 k$ & -6.31 & -67.8452 & -108.441 & -0.04 & -6.28 & 0.02 \\
\hline 16 & 81 & -5.79 & -68.5566 & -97.1257 & -0.80 & -5.44 & -0.35 \\
\hline 17 & $8 \mathrm{t}$ & -5.57 & -67.2658 & -102.789 & -0.64 & -4.75 & -1.18 \\
\hline 18 & $8 \mathrm{~m}$ & -5.11 & -55.2368 & -103.655 & -0.81 & -4.15 & -1.15 \\
\hline 19 & $8 d$ & -3.86 & -62.1285 & -105.235 & -0.8 & -5.85 & -1.21 \\
\hline 20 & 80 & -3.63 & -69.23 & -110.249 & -2.01 & -4.56 & -1.07 \\
\hline \multicolumn{8}{|c|}{ Standards were taken } \\
\hline \multicolumn{2}{|c|}{ Norfloxacin } & -7.29 & -54.2302 & -83.0725 & -1.68 & -4.14 & -1.04 \\
\hline
\end{tabular}

indicated that flouroquinolone derivatives showed similar or potent activity against $S$. aureus, $S$. pneumonia, E. coli, and $P$. aeruginosa as compared with norfloxacin.

3.3. Molecular Docking Studies of Derivatives. To understand the interaction of all the synthesized molecules $(\mathbf{8 a}-\mathbf{8 t})$ with topoisomerase II DNA gyrase enzymes, the crystal structure of topoisomerase II was downloaded from Protein Data Bank (PDB ID: 2XCT) and the molecular docking studies were performed using the Glide program. The protein ligand interaction plays a significant role in structural based drug designing. In this approach, $\mathrm{H}$-bonding, Glide energy score, Emodel, and $G$-score are kept as a support for the present work.

The preferred ligands $\mathbf{8 g}, \mathbf{8 i}, \mathbf{8 e}, \mathbf{8 c}, \mathbf{8 s}, \mathbf{8 q}, \mathbf{8 h}, \mathbf{8 n}$, and $\mathbf{8 a}$ had a $G$-score value of -8.51 to -7.43 . The $G$-score values of the above mentioned standard compound (norfloxacin) were comparatively lesser than their analogues with the scoring of -7.29 .

The minimum Glide energy required for the formation of complex between ligand and the receptor indicates excellent binding affinity. Very low energy indicates that the ligand is buried in the cavity of the receptor [17]. The Glide binding energy of the fluroquinolone analogues as ligands was found to be -54.38 to -74.83 . Standard compounds such as norfloxacin possessed Glide energy -54.23 .
In the present investigation, the nine fluoroquinolone analogues ligands had high quality binding affinity with the enzyme. Hydrogen bond interaction can act either as antagonist or as agonist for a ligand with receptor. On analyzing the docking results, all the seven compounds had $\mathrm{H}$-bond interactions with the receptor indicating that the ligand binding affinity increased together with the number of the H-bond interactions. All nine ligands had a significant number of $\mathrm{H}$-bond interactions with the enzyme.

Emodel had a more significant weighting of the force field components (electrostatic and van der Waals energies), which made it well suited for comparing conformers. Therefore, Glide uses Emodel to pick the "best" pose of a ligand (pose selection) and then ranks these best poses against one another with Glide score. A low Emodel value indicates a good binding affinity between protein and ligand. Glide Emodel values of -119.918 to -84.85 , for the nine best fluroquinolone analogues, were calculated (Table 3 ) indicating that these compounds engage in energetically more favourable interactions than norfloxacin (Emodel score -83.0725 , Table 3 ) within the active site.

From the docking study we predicted that fluoroquinolone analogues $(\mathbf{8 g}, 8 \mathbf{i}, 8 \mathbf{e}, 8 \mathbf{c}, 8 \mathbf{s}, 8 \mathbf{8}, \mathbf{8 h}, \mathbf{8 n}$, and $8 \mathbf{a})$ possess better antibacterial activity than the standard drugs by having good binding affinity with target protein and it could be used as potential drug as antimicrobial. Amongst all the docked compounds, compound $\mathbf{8 g}$ shows good binding 

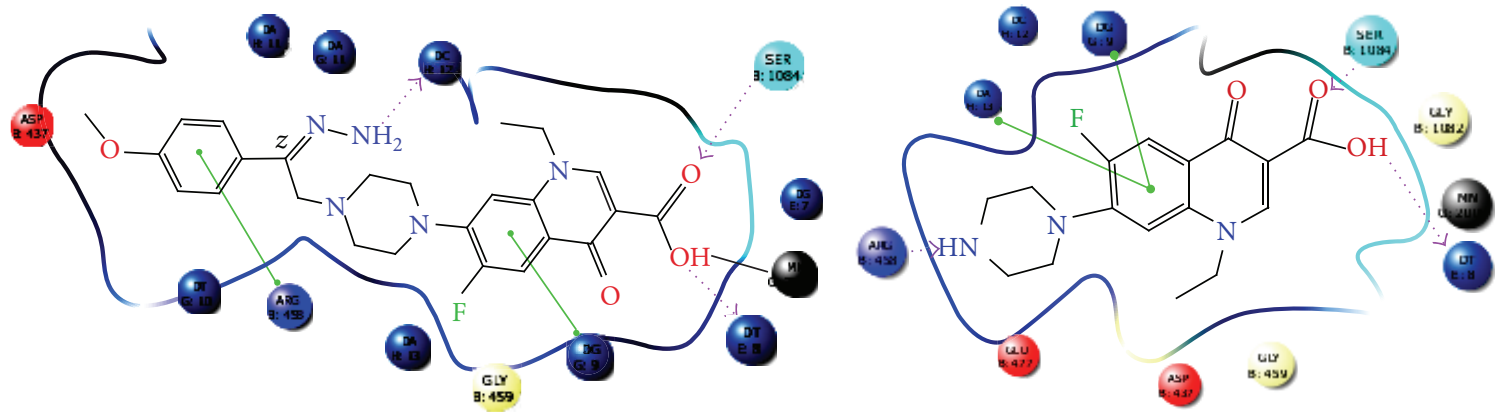

Charged (negative)
Charged (positive)
Polar
Hydrophobic
Glycine
- Metal
Water
Hydration site

(a)

$$
\begin{aligned}
\otimes & \text { Displaced hydration site } \\
\longrightarrow & \pi \text { - } \pi \text { stacking } \\
\longrightarrow & \pi \text {-cation } \\
\longrightarrow & \text { H-bond (backbone) } \\
\cdots & \text { H-bond (side chain) } \\
\longrightarrow & \text { Metal coordination } \\
- & \text { Salt bridge } \\
& \text { Solvent exposure }
\end{aligned}
$$
- Charged (negative)
- Charged (positive)
O Polar
- Hydrophobic
Glycine
- Metal
(3) Water
Hydration site

$$
\begin{aligned}
\otimes & \text { Displaced hydration site } \\
\longrightarrow & \pi-\pi \text { stacking } \\
\longrightarrow & \pi \text {-cation } \\
\longrightarrow & \text { H-bond (backbone) } \\
\cdots & \text { H-bond (side chain) } \\
\longrightarrow & \text { Metal coordination } \\
\longrightarrow & \text { Salt bridge } \\
& \text { Solvent exposure }
\end{aligned}
$$

(b)

FIGURE 1: 2D interaction diagram for the complex protein-ligand: (a) after docking interaction between 2XCT and $\mathbf{8 g}$ and (b) interaction between 2XCT and norfloxacin.

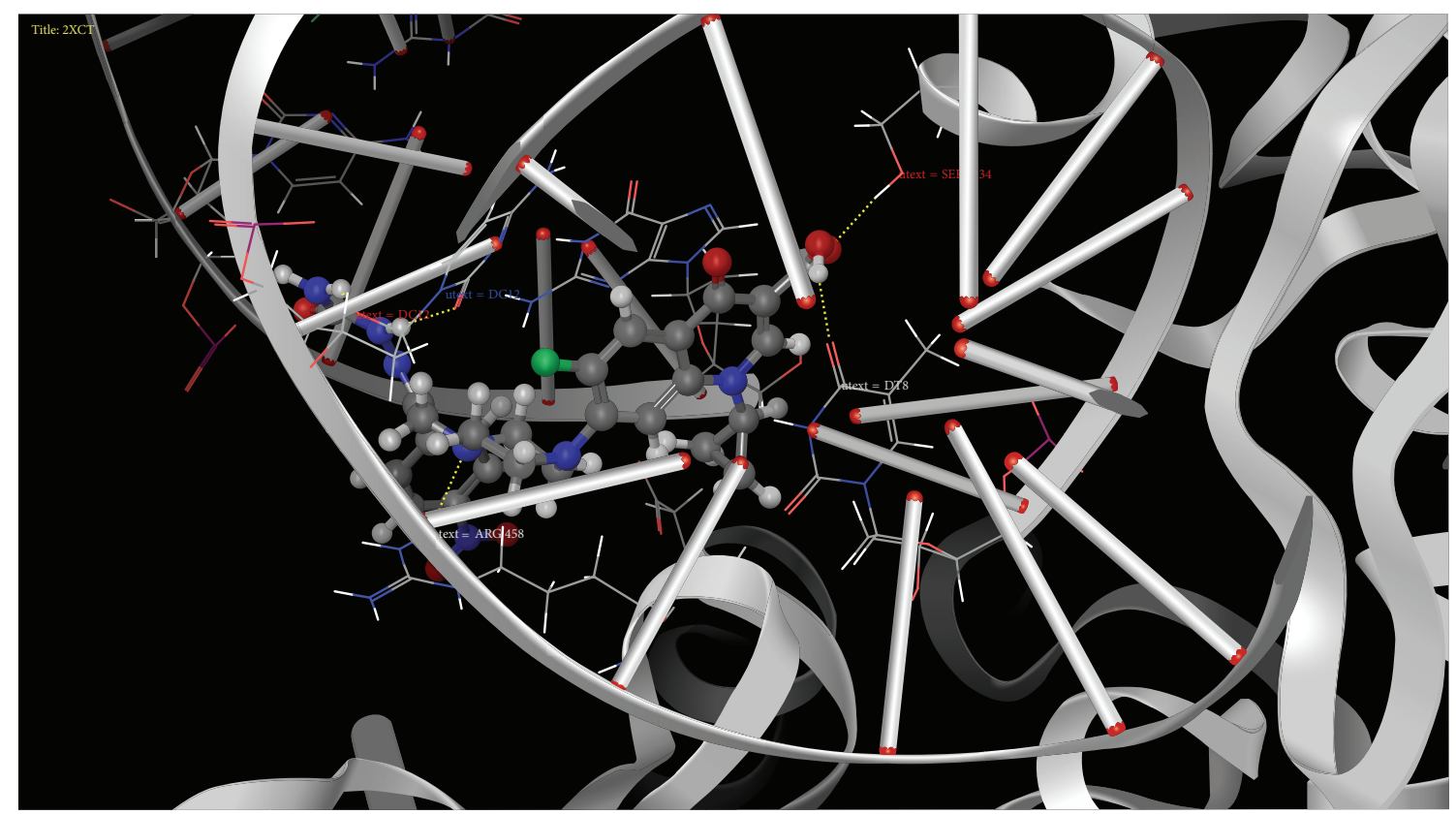

FIGURE 2: H-bond interactions between target protein 2XCT and studied compounds. Binding interaction of docked compound (8g) with topoisomerase II DNA gyrase enzymes.

affinity and interaction with topoisomerase II DNA gyrase enzymes (2XCT) with reference to norfloxacin shown in Figures 1 and 2.

\section{Conclusion}

In summary, a new series of novel 1-ethyl-6-fluoro-4oxo-7-\{4-[2-(4-substituted phenyl)-2-(substituted)-ethyl]-1piperazinyl $\}$-1,4-dihydroquinoline-3-carboxylic acid derivatives were synthesized and characterized by FT-IR, ${ }^{1} \mathrm{HNMR}$, and high resolution mass (HRMS-EI) spectral analyses. All the molecules were studied for their interactions with topoisomerase II DNA gyrase enzymes by molecular docking protocol. Among the tested molecules, compound $\mathbf{8 g}$ exhibited a good Glide score value of -8.51 and Glide energy -54.38 with Emodel value of -105.2 . In vitro antibacterial activity of the tested compounds shows improved activity against all the microorganisms used. In particular, compound $\mathbf{8 g}$ exhibits marked activity against two microorganisms. The results of antibacterial activity are supported by docking analysis. The 
nice docking scores of $\mathbf{8 g}, \mathbf{8 h}, \mathbf{8 i}, \mathbf{8 n}$, and $\mathbf{8 q}$ reveal that these compounds are well accommodated in active site of enzyme and the binding pattern of compounds $\mathbf{8 g}, \mathbf{8 h}, \mathbf{8 i}, \mathbf{8 n}$, and $\mathbf{8 q}$ showed that they strongly interact within the active site of topoisomerase II DNA gyrase enzymes (2XCT).

\section{Conflict of Interests}

The authors declare that there is no conflict of interests regarding the publication of this paper.

\section{Acknowledgment}

The authors are very thankful to Dr. R. H Parikh, Principal, Ramanbhai Patel College of Pharmacy, Changa, and Charotar University of Science and Technology (CHARUSAT), for providing the research facilities.

\section{References}

[1] Z. Yang, Y. Kitano, K. Chiba et al., "Synthesis of variously oxidized abietane diterpenes and their antibacterial activities against MRSA and VRE," Bioorganic \& Medicinal Chemistry, vol. 9, no. 2, pp. 347-356, 2001.

[2] C. P. Ravi, D. Premnath, and S. Vasanth Kumar, "Design, synthesis, molecular docking and antibacterial evaluation of novel $\mathrm{N}$-(6, 11-dioxo-dihydro-5h-benzo [B] carbazol-2yl) benzamide derivatives as potent antibacterial agents," International Journal of Pharmacy and Pharmaceutical Sciences, vol. 6, no. 6, pp. 244249, 2014.

[3] Z. Yu, G. Shi, Q. Sun et al., "Design, synthesis and in vitro antibacterial/antifungal evaluation of novel 1-ethyl-6-fluoro1,4-dihydro-4-oxo-7(1-piperazinyl)quinoline-3-carboxylic acid derivatives," European Journal of Medicinal Chemistry, vol. 44, no. 11, pp. 4726-4733, 2009.

[4] M. L. D. Souza, V. F. Ferreira, B. A. Lucero, and C. R. B. Gomes, "Synthesis several 1-[(2-hydroxy-ethoxy)methyl]-3-carbethoxy-4 $(1 \mathrm{H})$ quinolones and 1-[(2-hydroxy-ethoxy)methyl]4(1H)quinolone-3-carboxylic acids as quinolonic acyclovir analogues and evaluated anti-HSV-1 activity against herpes simplex virus type 1(HSV-1)," Bioorganic And Medicinal Chemistry Letters, vol. 16, pp. 1010-1014, 2006.

[5] O. Tabarrini, S. Massari, D. Daelemans et al., "Structure-activity relationship study on anti-HIV 6-desfluoroquinolones," Journal of Medicinal Chemistry, vol. 51, no. 17, pp. 5454-5458, 2008.

[6] W. Wang, Q. D. You, Z. Y. Li, and Y. Q. Zou, "Design, synthesis and antitumor activity of 3-substituted quinolone derivatives (I)," Chinese Chemical Letters, vol. 19, no. 12, pp. 1395-1397, 2008.

[7] N. Srivastava, A. Kumar, and A. Mehrotra, "A facile synthesis of antibacterial norfloxacin by microwave irradiations," International Journal of Chemistry, vol. 34, no. 2, pp. 1167-1171, 2012.

[8] G. V. Reddy, S. R. Kanth, D. Maitraie et al., "Design, synthesis, structure-activity relationship and antibacterial activity series of novel imidazo fused quinolone carboxamides," European Journal of Medicinal Chemistry, vol. 44, no. 4, pp. 1570-1578, 2009.

[9] S. K. Sahu, S. N. Pandeya, and A. K. Pathak, "Synthesis and antimicrobial evaluations of N-substituted piperazinyl Schiff bases of gatifloxacin," Medicinal Chemistry and Drug Discovery, vol. 3, no. 1, pp. 1-10, 2012.
[10] Prime, Version 3.6, Schrödinger, LLC, New York, NY, USA, 2014.

[11] R. A. Friesner, R. B. Murphy, M. P. Repasky et al., "Extra precision glide: docking and scoring incorporating a model of hydrophobic enclosure for protein-ligand complexes," Journal of Medicinal Chemistry, vol. 49, no. 21, pp. 6177-6196, 2006.

[12] Ligprep, Version 3.0, Schrödinger, LLC, New York, NY, USA, 2014.

[13] Maestro, Version 9.7, Schrödinger LLC, New York, NY, USA, 2014.

[14] K. S. Watts, P. Dalal, R. B. Murphy, W. Sherman, R. A. Friesner, and J. C. Shelley, "ConfGen: a conformational search method for efficient generation of bioactive conformers," Journal of Chemical Information and Modeling, vol. 50, no. 4, pp. 534-546, 2010.

[15] C. Monika, District Laboratory Practice in Tropical Countries. Part-II, Manual, Cambridge University Press, Cambridge, UK, 2nd edition, 2006.

[16] E. J. Baron and S. M. Finegold, Bailey Scott's Diagnostic Microbiology, The C.V. Mosby Company, St. Louis, Mo, USA, 11th edition, 2002.

[17] P. Daisy, P. Vijayalakshmi, C. Selvaraj, S. K. Singh, and K. Saipriya, "Targeting multidrug resistant Mycobacterium tuberculosis HtrA2 with identical chemical entities of fluoroquinolones," Indian Journal of Pharmaceutical Sciences, vol. 74, no. 3, pp. 217-222, 2012. 


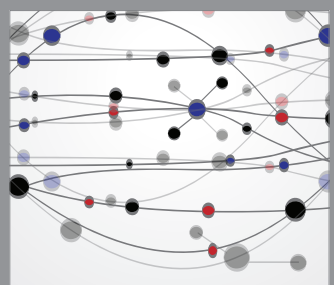

The Scientific World Journal
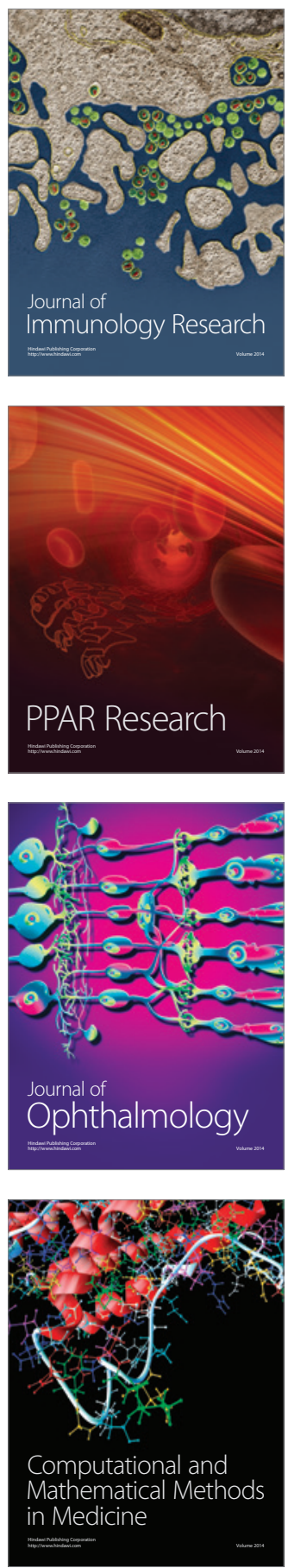

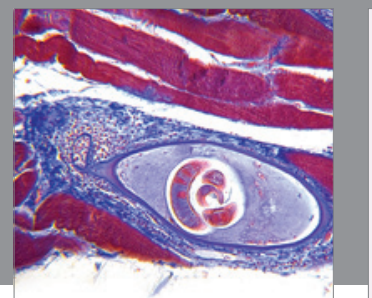

Gastroenterology

Research and Practice
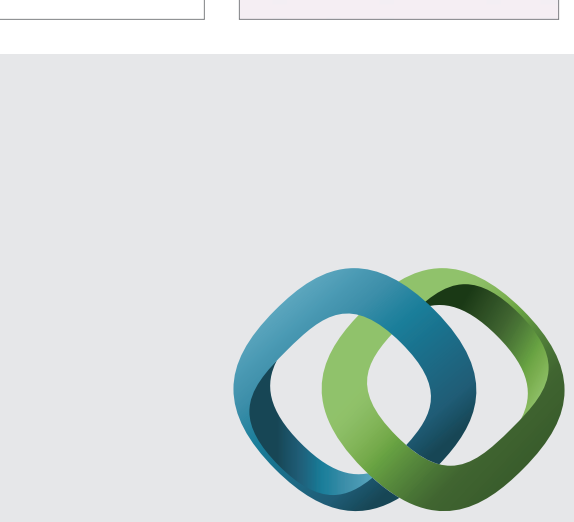

\section{Hindawi}

Submit your manuscripts at

http://www.hindawi.com
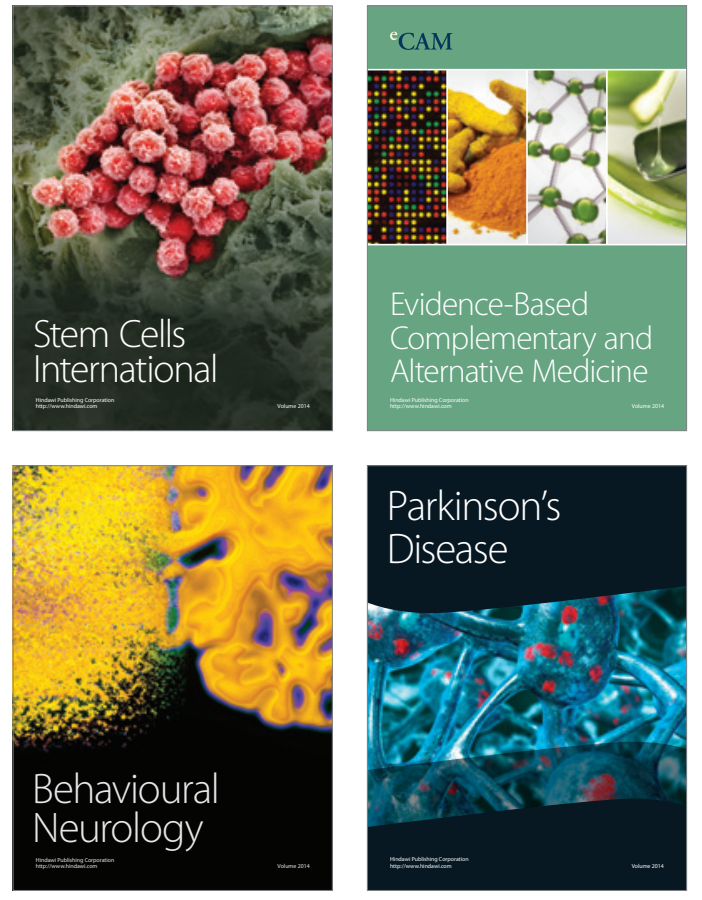
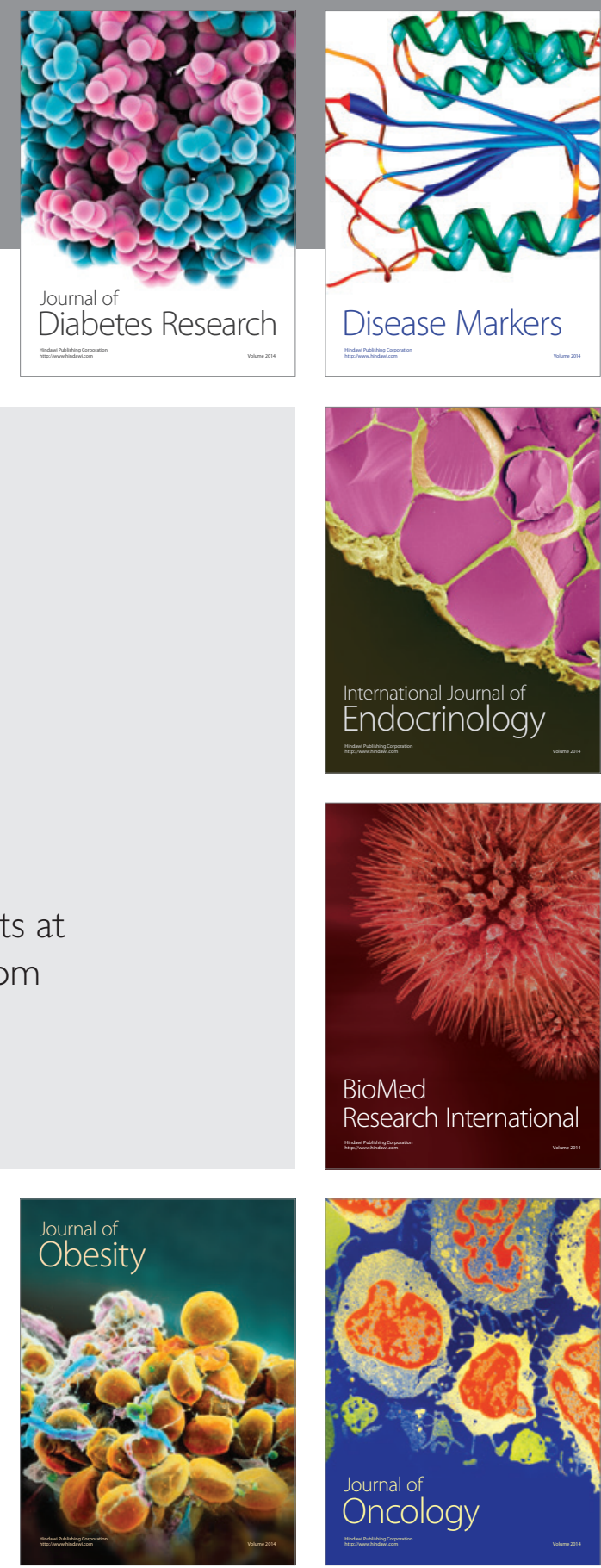

Disease Markers
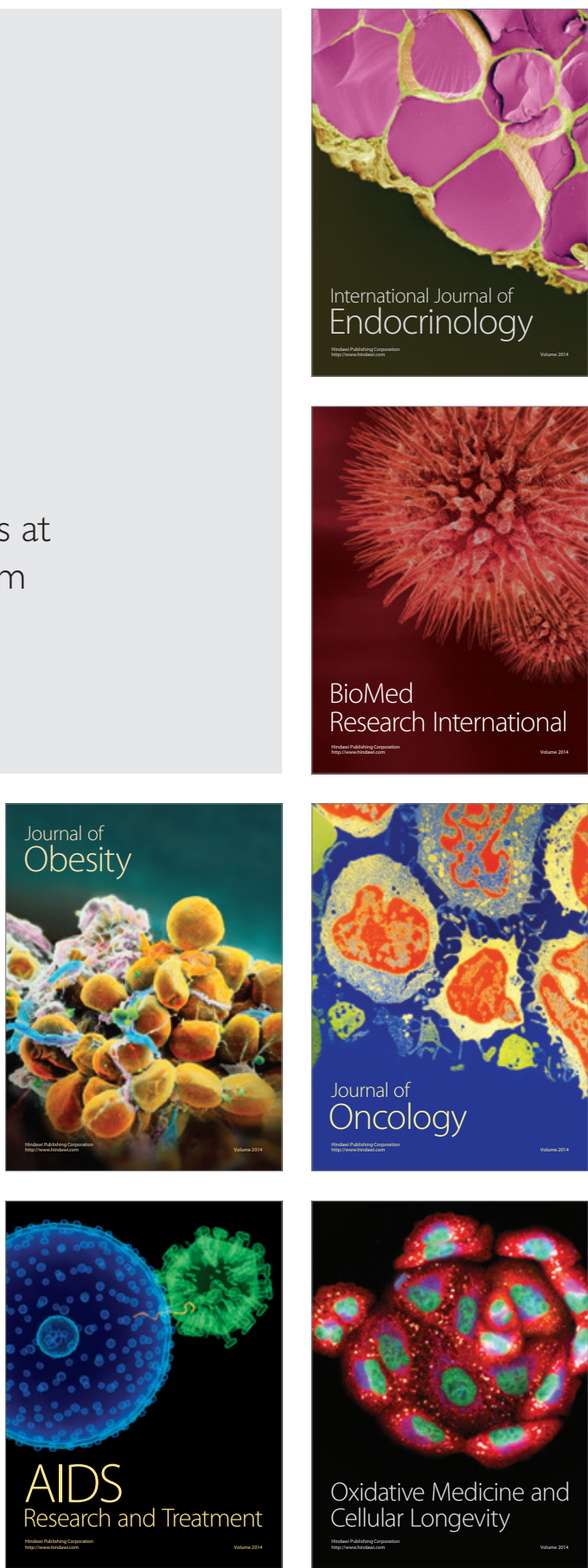